Check for updates

Cite this: RSC Adv., 2018, 8, 36133

\title{
Inhibition of biofilm formation and quorum sensing mediated phenotypes by berberine in Pseudomonas aeruginosa and Salmonella typhimurium
}

\author{
Jamuna Bai Aswathanarayan and Ravishankar Rai Vittal (D) *
}

Quorum sensing is involved in biofilm formation and modulates virulence factor production in pathogenic bacteria. Quorum sensing inhibitors can be used as novel intervention strategies for attenuating bacterial pathogenicity. Berberine is an isoquinoline alkaloid with pharmacological properties. The present study investigated the sub-inhibitory concentrations of berberine for inhibiting biofilm formation and quorum sensing regulated phenotypes in the bacterial pathogens Pseudomonas aeruginosa PA01 and Salmonella enterica serovar Typhimurium. Berberine inhibited quorum sensing regulated violacein production in $C$. violaceum. It reduced the pigment production in the wild type strain at $1.6 \mathrm{mg} \mathrm{mL}^{-1}$ by $62.67 \%$. In the opportunistic pathogen, $P$. aeruginosa PA01, at sub-MIC, it showed significant antibiofilm activity in by reducing biomass by $71.70 \%(p<0.05)$. It prevented biofilm formation and inactivated biofilm maturation in bacterial pathogens at the concentration ranging from 0.019 to $1.25 \mathrm{mg} \mathrm{mL}^{-1}$. In silico studies showed that berberine interacted with the quorum sensing signal receptors, LasR and RhIR. Furthermore, its antiinfective properties in S. Typhimurium were studied. At sub-inhibitory concentrations of $0.019 \mathrm{mg} \mathrm{mL}^{-1}$, it reduced biofilm formation in S. Typhimurium by $31.20 \%$. It significantly prevented invasion and adhesion of Salmonella invasion in the colonic cell, HT 29 by $55.37 \%$ and $54.68 \%$, respectively. It was capable of reducing in vivo virulence in Caenorhabditis elegans infected with Salmonella at $0.038 \mathrm{mg}$ $\mathrm{mL}^{-1}$ by $65.38 \%$. Our results suggest that berberine, previously recognised for its antimicrobial activity, could find potential application as an anti-biofilm and anti-infective agent based on its quorum sensing inhibitory activity.

Received 30th July 2018

Accepted 12th October 2018

DOI: $10.1039 / \mathrm{c} 8 \mathrm{ra06413j}$

rsc.li/rsc-advances

\section{Introduction}

Quorum sensing is a cell density dependent mechanism used by bacteria to regulate gene expression. It is an essential component of biofilm physiology, as biofilms have a high concentration of bacterial cells. ${ }^{1}$ Quorum sensing or cell to cell communication is utilized by bacteria for biofilm initiation, maturation and dispersal and also for regulating metabolic activity and population density within the biofilm. ${ }^{2}$ Therefore, anti-biofilm strategies can be developed based on inhibition or interruption of the quorum sensing mechanism in bacteria. ${ }^{3}$

Apart from its role in biofilm formation, the quorum sensing mechanism is known to modulate virulence in pathogenic bacteria at molecular levels. ${ }^{4}$ Therefore, potential quorum sensing inhibitors could be developed as anti-infective agents against pathogenic bacteria. The phytochemicals having structural analogy to the AHLs compete with the signaling molecules to bind to the quorum sensing receptors. Hence, these

Department of Studies in Microbiology, University of Mysore, Mysore, India. E-mail: raivittal@gmail.com compounds have the potential to be used as quorum sensing inhibitors. ${ }^{5}$ Phytochemicals such as furocoumarins have inhibited quorum sensing and biofilms in the pathogenic Vibrio harveyi, E. coli $\mathrm{O} 157: \mathrm{H} 7$, S. enterica sv Typhimurium and $P$. aeruginosa. Purified plant derived compounds including furocoumarins and bergamottin were shown to inhibit signal molecule production by $94.6-97.7 \%$ and modulate quorum sensing regulated phenotypes. ${ }^{6}$ Thus, compounds derived from plants and other dietary phytochemicals generally considered as safe can be screened for their effect on quorum sensing regulated phenotypes and further developed as quorum sensing inhibitors against the pathogens.

Berberine hydrochloride, a protoberberine alkaloid is a compound with various pharmacological effects including antimicrobial, antimalarial, anti-inflammatory, and anticancer activities. It has been used as a therapeutic agent in clinical applications due to its inherent low cytotoxicity. ${ }^{7}$ It is also widely used for the treatment of bacterial and fungal infections. At sub-MICs berberine inhibited MRSA biofilms in a concentration dependent manner. It has shown to inhibit MRSA amyloid fibril formation proving its efficacy as an agent for 
treating microbial-generated amyloid diseases. ${ }^{8}$ It was capable of inhibiting biofilms of Staphylococcus epidermidis, a clinically relevant pathogen involved in medical-device associated infections at sub-MICs. ${ }^{9}$ Similarly, berberine in combination with other antibiotics is known to reduce biofilm formation in clinical strains of $S$. aureus. It was capable of effectively downregulating the expression of the biofilm-associated sarA, cidA and icaA genes. ${ }^{10}$ Berberine has also inhibited biofilm formation and promoted biofilm dispersion in UTI causing Enterococcus faecalis. ${ }^{11}$ It is proved to be a good endodontic irrigant against multispecies biofilms of endodontic pathogens due to its bactericidal efficacy. ${ }^{12}$

In the present study, the phytochemical berberine belonging to the class isoquinoline alkaloids was studied at sub-inhibitory concentrations for the quorum sensing and biofilm inhibitory activity and anti-infective potential in Gram negative pathogens $P$. aeruginosa PA01 and S. enterica sv Typhimurium. The phytochemical was also tested for its ability to inhibit quorum sensing regulated phenotypes in biosensor strains of $C$. violaceum and $P$. aeruginosa PA01. The putative interaction of the compound with the quorum sensing signal receptors was analysed by computational studies. Further, its effect on virulence, invasion and pathogenicity in S. enterica sv Typhimurium was studied. The phytochemical berberine can be further developed into anti-biofilm and anti-infective agent based on its antiquorum sensing mechanism.

\section{Materials and methods}

\section{Bacterial strains and chemicals}

Chromobacterium violaceum (ATCC 12472), C. violaceum CV026, $P$. aeruginosa $\mathrm{PAO} 1$ and $P$. aeruginosa PAO1-JP2 were used for determining the quorum sensing inhibitory potential of the phytochemicals. C. violaceum (ATCC 12472) is a wild type strain whereas C. violaceum CV026 is a mutant, unable to produce signaling molecules but responds to short chain of AHLs. $P$. aeruginosa $\mathrm{PAO} 1$ is a wild type and $P$. aeruginosa $\mathrm{PAO} 1-\mathrm{JP} 2$ is lasI and rhII mutants unable to produce AHLs. The strains were cultured in Luria Bertani media at $28^{\circ} \mathrm{C}$. Salmonella enterica var typhimurium a clinical isolate was used for anti-infective study. The pathogen was grown and maintained in nutrient broth at $37{ }^{\circ} \mathrm{C}$. The nematode Caenorhabditis elegans used for antivirulence studies was grown in nematode growth media at $25^{\circ} \mathrm{C}$. E. coli OP50 was cultured in LB media at $37^{\circ} \mathrm{C}$.

Berberine chloride used in the study was purchased from Sigma-Aldrich (B251-5G, St. Louis, USA). Its purity is $>98 \%$. The QS signal molecule $N$-butanyl--L-homoserine lactone (C4-HSL) and QS inhibitor furanone C30 purchased from SigmaAldrich, India were used in the experiments.

\section{Determination of MIC}

The minimum inhibitory concentration of berberine was determined in the Mueller-Hinton broth using a broth microdilution method. ${ }^{13}$ The MIC of the phytochemical against the bacteria used in the assays were assessed in the concentration ranging from 1.250 to $0.019 \mathrm{mg} \mathrm{mL}^{-1}$. The MIC was recorded as the lowest concentration exhibiting complete inhibition of visible growth and confirmed by the addition of tetrazolium trichloride dye. All the experiments in the present study were performed at sub-MIC concentrations of the phytochemical.

\section{Quorum sensing inhibitory activity of berberine}

Inhibition of violacein production in C. violaceum. Quorum sensing inhibitory activity of the phytochemicals was quantified by measuring the inhibition of violacein production in C. violaceum (ATCC 12472) and C. violaceum CV026. ${ }^{14}$ LB broth supplemented with sub-inhibitory concentration of berberine was inoculated with $200 \mu \mathrm{L}$ of C. violaceum culture (WT) and incubated at $28{ }^{\circ} \mathrm{C}$. Violacein extraction was carried out from the phytochemical treated culture and control sample by adding $200 \mu \mathrm{L}$ of $10 \%$ SDS, vortexing for $15 \mathrm{~s}$ and incubated at room temperature for $5 \mathrm{~min}$. The cell lysate was treated with $900 \mu \mathrm{L}$ of water-saturated butanol ( $50 \mathrm{~mL}$ of $n$-butanol mixed with $10 \mathrm{~mL}$ distilled water), vortexed for $5 \mathrm{~s}$ and centrifuged at $13000 \mathrm{~g}$ for $5 \mathrm{~min}$. The absorbance of butanol phase containing violacein was measured at $585 \mathrm{~nm}$. The percentage inhibition of violacein production was calculated.

Similar studies were performed with C. violaceum CV026 biosensor strain. Overnight grown C. violaceum CV026 culture was inoculated in LB medium supplemented with C4-HSL and the sub-inhibitory concentration of berberine. After incubation, the violacein was extracted and quantified as previously described. The quorum sensing inhibitory activity was indicated by reduction in violacein production.

Inhibition of swimming, swarming and twitching motility. Motility assay was carried out by adding sub-inhibitory concentration of berberine into the motility media and inoculating with $5 \mu \mathrm{L}$ of bacterial broth culture $\left(10^{8} \mathrm{CFU} \mathrm{mL}^{-1}\right)$. The swim plates contained $0.3 \%$ agar and was inoculated with bacterial culture directly into the center of the LB media to evaluate motility in the semisolid agar. Swarm plates were prepared with $0.5 \%$ agar and inoculum placed in the center of agar surface to evaluate bacterial motility across the surface. Twitching motility plates contained $1 \%$ agar and bacteria culture was inoculated deep into the media. The diameters of the all three motility zones were measured after incubation at $37^{\circ} \mathrm{C}$ for $24 \mathrm{~h} .{ }^{15}$

\section{Antibiofilm activity of berberine}

The biofilm inhibitory activity of berberine was studied in $P$. aeruginosa PAO1 and $S$. enterica sv Typhimurium. Its ability to inhibit biofilm formation at 0.5 MIC was assessed by the crystal violet assay. ${ }^{16}$ In brief, $100 \mu \mathrm{L}$ of 0.5 MIC equivalent phytochemical and $100 \mu \mathrm{L}$ of bacteria cultures (absorbance $=0.4$ at $600 \mathrm{~nm}$ ) grown in tryptic soy broth was added to individual wells of a sterile flat-bottomed 96-well polystyrene microtitre plates microlitres. The plates were incubated for $24 \mathrm{~h}$ at $37^{\circ} \mathrm{C}$ for biofilm formation. The experiment was carried out in triplicates.

The biofilm inhibition by phytochemical was confirmed as a result of anti-QS property by using the control strain P. aeruginosa PAO1-JP2. Overnight grown culture was grown in TSB was 
adjusted to get $1.5 \times 10^{8} \mathrm{CFU} \mathrm{mL} \mathrm{m}^{-1}$ and $190 \mu \mathrm{L}$ culture was added to individual wells of a sterile 96 well microtiter plate. Further, the signaling molecules $(10 \mu \mathrm{L}$ of $10 \mu \mathrm{M}$ 3-oxo-C12 HSL or C4-HSL) were added to each well with sub-MIC of berberine. Control wells contained only media, cells without AHLs, cells with AHLs but without berberine and cells with berberine without AHLS. After incubation at $37{ }^{\circ} \mathrm{C}$ for $24 \mathrm{~h}$ the biofilm formation was determined by crystal violet technique. ${ }^{17}$

\section{Fluorescence microscopy}

Biofilms were studied using fluorescence microscopy techniques. ${ }^{18}$ Bacterial cells $\left(10^{8} \mathrm{CFU} \mathrm{mL}^{-1}\right)$ grown in tryptic soy broth was dispensed into sterile Petriplates $(90 \mathrm{~mm})$ containing sterilized cover slips $(22 \times 48 \mathrm{~mm})$ treated with and without sub-MIC of the phytochemicals. The plates were incubated at $37{ }^{\circ} \mathrm{C}$ under static conditions for the development of biofilms. After the $24 \mathrm{~h}$ incubation period, the cover slips were stained with $20 \mu \mathrm{L}$ acridine orange dye (1\%) (Sigma-Aldrich, Switzerland). The stained glass cover slips were incubated for $30 \mathrm{~min}$ under dark condition, rinsed with sterile water to remove excess stain, air dried and further visualized with fluorescence microscopy at $40 \times$ air objective and (Axio Scope-Carl Zeiss, Germany). The software AxioVision 4.6 was used for digital processing of the images (Carl Zeiss, Germany).

\section{Salmonella - adhesion and invasion assays}

HT 29 a human colon cell line was procured from NCCS, Pune (India). The viability of cells in presence of berberine was tested by 3-(4,5-dimethyl-2-thiazolyl)-2,5-diphenyl-2H-tetrazolium bromide (MTT) assay. ${ }^{19}$ The assay was performed using EzCount MTT Cell assay Kit (Himedia, India). Cells were cultured in DMEM with 10\% FBS and 1\% penicillin-streptomycin at $5 \% \mathrm{CO}_{2}$ at $37{ }^{\circ} \mathrm{C}$. On reaching $90 \%$ confluency, cells were seeded in 96-well plates. $200 \mu \mathrm{L}$ of diluted cell suspension $\left(1 \times 10^{5}\right.$ cell per $\left.\mathrm{mL}\right)$ was added to each well. The cells were incubated for $24 \mathrm{~h}$. After the incubation period, different concentrations of berberine 0.5 to $20 \mu \mathrm{L}$ were added to the cells, followed by incubation for $24 \mathrm{~h} .20 \mu \mathrm{L}$ of MTT dye $\left(5 \mathrm{mg} \mathrm{mL}^{-1}\right)$ was added to each well. The plates were gently shaken and incubated for $4 \mathrm{~h}$. The media containing MTT dye solution was removed, $200 \mu \mathrm{L}$ of solubilizing solution was added to solubilize the formed formazan. The absorbance was measured at $570 \mathrm{~nm}$. Cytotoxicity was calculated and expressed as percentage inhibition.

The adhesion and invasion assay were performed in HT 29 cell line using $S$. Typhimurium. ${ }^{20}$ Briefly, the cell adhesion involved centrifuging overnight grown $S$. Typhimurium cultures resuspended it in DMEM to obtain $1 \times 10^{8} \mathrm{CFU} \mathrm{\textrm {mL } ^ { - 1 }}$. The bacterial culture and different concentrations of phytochemicals were added to monolayers of HT29 cells grown in 6 well microtitre plates and incubated for $1 \mathrm{~h}$ at $37{ }^{\circ} \mathrm{C}$. The wells were washed thrice with PBS. The cells were lysed using $1 \%$ Triton X100 on incubation at room temperature for $20 \mathrm{~min}$. The lysated suspensions were serially diluted and $100 \mu \mathrm{L}$ of dilution was plated on trypticase soy agar. After incubation for $24 \mathrm{~h}$ at $37^{\circ} \mathrm{C}$, the bacterial colonies were used to calculate the adhesion rate.
Similarly, in the invasion assay the HT29 cell monolayers were washed once with PBS after $1 \mathrm{~h}$ of incubation with $S$. Typhimurium resuspended in DMEM with different concentrations of berberine. Further, it was incubated for 30 min with $1 \mathrm{~mL}$ DMEM supplemented with $100 \mu \mathrm{g} \mathrm{mL}^{-1}$ gentamicin to kill extracellular bacteria. The wells were washed with PBS and lysed with $1 \%$ Triton X-100. The suspension was serially diluted and plated on TSA. The number of invaded or intracellular bacteria was calculated by counting the colonies on TSA plates.

\section{In vivo virulence of $S$. Typhimurium in Caenorhabditis elegans (N2) model}

$S$. Typhimurium was grown overnight in nutrient broth at $37^{\circ} \mathrm{C}$. The overnight bacterial culture was re-suspended in $\mathrm{S}$ Basal liquid assay media and $800 \mu \mathrm{L}$ of the bacterial suspension was aliquoted into each well of a 96-well flat-bottomed plate. Berberine at 0.019 and $0.038 \mathrm{mg} \mathrm{mL}^{-1}$ and furanone $(1 \mu \mathrm{M})$ was added to the wells. Twenty L4-staged worms were added to each well and were incubated at $23{ }^{\circ} \mathrm{C}$ for $24 \mathrm{~h}$. Each well was examined with a microscope to observe the infection phenotype in C. elegans. Infected worms were also transferred to unseeded NGM plates to verify the phenotype. Worms were considered paralysed when neither body twitching nor pharyngeal pumping could be observed. Percentage of paralysed worms were calculated to determine anti-virulence effect of $S$. Typhimurium. ${ }^{21}$

\section{Molecular docking studies}

The crystal structures of $P$. aeruginosa LasR (PDB ID: 2UV0) as well as a homology model of $P$. aeruginosa RhlR were employed for setting-up molecular docking protocols. Prior to this, the protein coordinates were optimized through suitable bondorder assignments, water molecules elimination, addition of hydrogen atoms, internal hydrogen bonds refinement, restrained minimization etc. through OPLS 2005 force-field. Docking grids were generated within $20 \AA$ from the ligand binding site (or equivalent site in case of the homology model). The small molecules (compounds) were energy minimized through LigPrep and then docked in to the generated grid employing extra precision (XP) mode of Glide 6.2. All docked molecules were finally processed through a post-docking minimization protocol of the same software. The images were prepared using PyMOL 1.0.

\section{Homology modeling}

The target sequence of full-length $P$. aeruginosa RhlR (1-241) was retrieved from UniProt (Acc. code: P54292). This was further subjected to homology model building employing 'knowledgebased' protocol of Prime 3.5. The generated model was further optimized through MacroModel 10.3 in a simulated aqueous environment and employing OPLS 2005 force-field. The final model was observed to have a reasonable Ramachandran plot and only few atypical side-chain conformations. All software names mentioned above are products of Schrödinger LLC. ${ }^{22}$ 


\section{Statistical analysis}

All the experiments were performed in triplicates and the data were reported as means + standard deviation. Student's $t$ test was used to analyze the differences between control and test. One-way analysis of variance (ANOVA) was used in analyzing differences in the different assays. The $p$ values $<0.05$ were considered statistically significant. The statistical analysis was carried out using IBM SPSS Statistics 18.

\section{Results and discussion}

\section{Determination of minimum inhibitory concentration}

In the present study, the phytochemical berberine was studied for the quorum sensing modulatory and anti-biofilm activity. The MIC of the phytochemical against the tested bacteria were determined at a concentration ranging from 0.019 to $2.50 \mathrm{mg}$ $\mathrm{mL}^{-1}$ (Table 1). Sub-inhibitory concentration of the phytochemicals were used in the study as they are known to inhibit quorum sensing regulated phenotypes, without affecting the growth of the bacteria. The absorbance of the growth of the phytochemical treated and control bacteria did not show any significant difference.

\section{Quorum sensing inhibitory activity of berberine}

Inhibition of violacein production. The quorum sensing inhibitory potential of berberine was screened using the biosensor bacteria $C$. violaceum. Violacein pigment production in C. violaceum (WT) is regulated by the CviIR-an

Table 1 Determination of MIC of berberine against bacterial pathogens

\begin{tabular}{ll}
\hline Pathogens & $\mathrm{MIC}\left(\mathrm{mg} \mathrm{mL}^{-1}\right)$ \\
\hline C. violaceum & 2.50 \\
C. violaceum CV026 & 2.50 \\
$P$. aeruginosa PA01 & 1.250 \\
$P$. aeruginosa PA01-JP2 & 0.156 \\
$S$. Typhimurium & 0.076
\end{tabular}

acylhomoserine lactone based quorum sensing system. The compounds capable of inhibiting violacein production at sub inhibitory concentration without reducing the growth of the bacteria are considered potential quorum sensing inhibitors. These can interfere with the CviR-AHL complex and prevent expression of the vio genes required for the production of violacein. Similarly, in C. violaceum CV026 the biosensor strain produces violacein only in presence of exogenous signal molecules such as C4-HSL. The QS inhibitors can prevent violacein production in $C$. violaceum CV026 even in presence of the autoinducers. ${ }^{23}$ Berberine was capable of inhibiting production of the pigment violacein in both the wildtype and mutant of $C$. violaceum at the tested concentrations (Fig. 1). Berberine reduced violacein production in the wildtype by $62.67 \%$ at

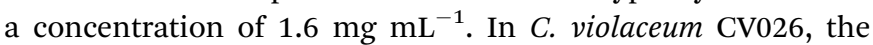
pigment production was reduced by $73.52 \%$ at the same concentration. Previously, it has been shown that cinnamaldehyde at minimal concentration was capable of inhibiting acyl homoserine lactone (3-oxo-C6-HSL and 3-oxo-C12-HSL) and autoinducer 2 mediated quorum sensing. ${ }^{24}$ Similarly, curcumin at concentrations ranging from $25-100 \mu \mathrm{g} \mathrm{mL}^{-1}$ has been shown to inhibit hexanoyl homoserine lactone mediated quorum sensing in C. violaceum and formation of biofilms by uropathogens..$^{25}$ However, there are no reports of the quorum sensing inhibitory potential of the phytochemical berberine. Phytochemicals having structural homology to the acyl homoserine lactones may act as competitive inhibitors and prevent the signal reception or decrease the expression of the signal synthases, and thus inhibit expression of quorum sensing regulated phenotypes. ${ }^{26}$

Inhibition of swimming, swarming and twitching motility. Berberine inhibited both swimming and swarming motility in $P$. aeruginosa PA01. It had no inhibitory effect on twitching motility in P. aeruginosa PA01 (Fig. 2). Berberine did not interfere with swimming, swarming and twitching motility in $S$. enterica sv Typhimurium. It significantly inhibited swarming motility in $P$. aeruginosa PA01 by $33.34 \%$ at $0.625 \mathrm{mg} \mathrm{mL} \mathrm{m}^{-1}$. Berberine exhibited maximum inhibition in swimming motility

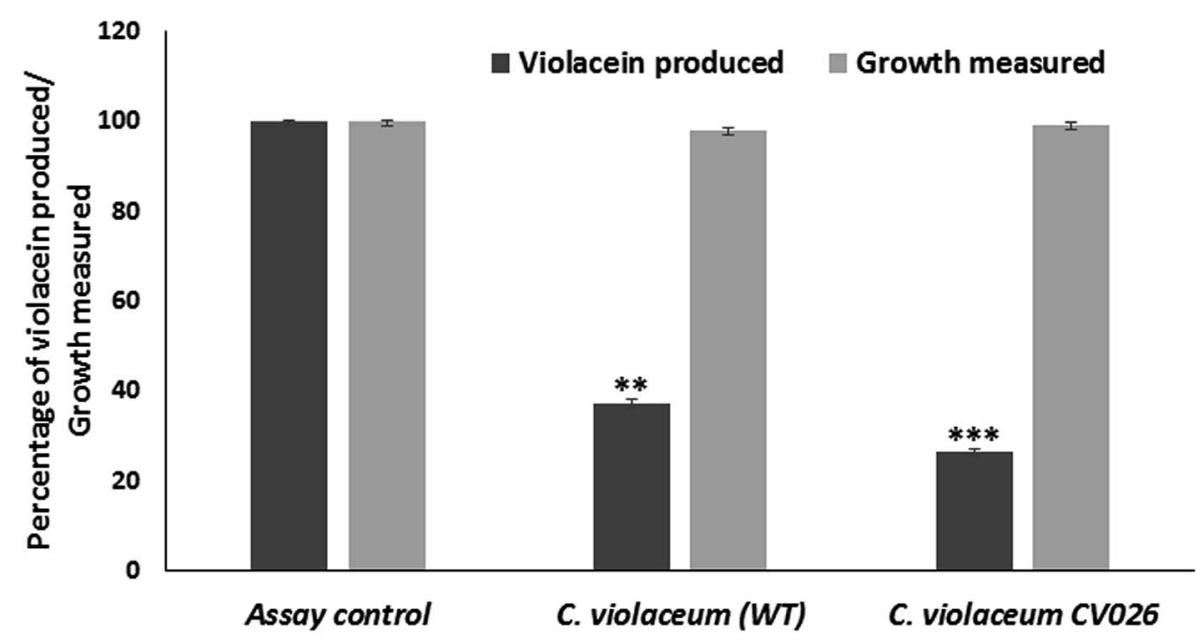

Fig. 1 Effect of sub-inhibitory concentration of berberine on quorum sensing mediated violacein production in C. violaceum (wild type) and C. violaceum CV026. 

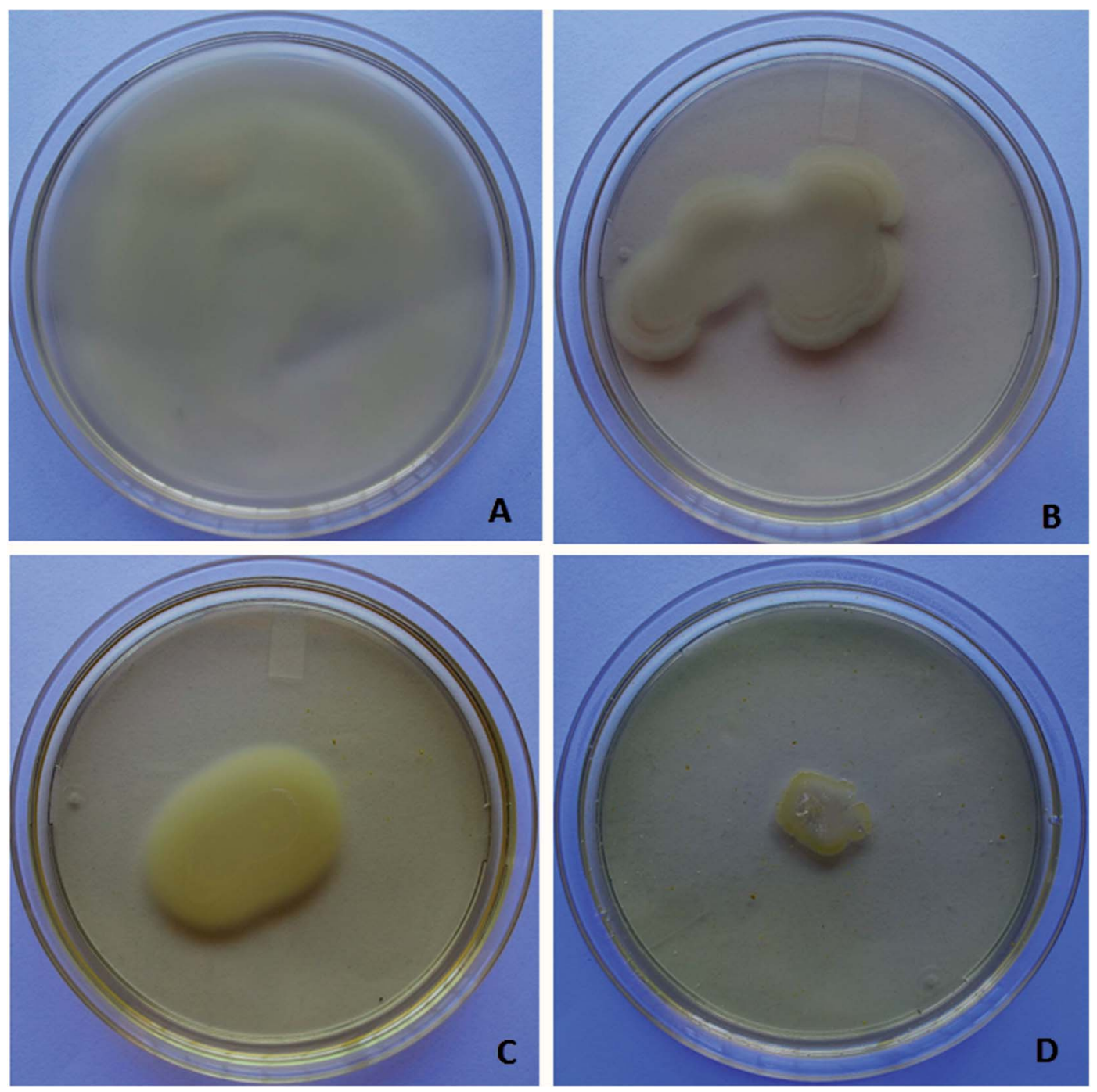

Fig. 2 Inhibitory effect of berberine on swimming and swarming motility in P. aeruginosa PA01. Swimming (A) and swarming motility (B) control in P. aeruginosa PA01; swimming (C) and swarming motility (D) in P. aeruginosa PA01 at sub-inhibitory concentration of berberine.

at $0.312 \mathrm{mg} \mathrm{mL}{ }^{-1}$. The antibacterial activity of the phytochemical berberine has been asserted previously. However, there are no reports to date on its effect on virulence factor production in pathogenic bacteria. In bacteria, motility is required for microbial surface colonization, biofilm formation and pathogenesis. Motility is also known to be regulated by quorum sensing mechanism in $P$. aeruginosa.${ }^{15}$ Berberine exerted inhibitory effect on swimming and swarming motility in $P$. aeruginosa PA01 at sub-MIC. Quorum sensing inhibitory compounds such as halogenated furanones are known to inhibit swarming motility in pathogenic bacteria. ${ }^{27}$ The phytochemicals capable of affecting bacterial motility can be used to prevent bacterial surface colonization.

\section{Antibiofilm activity of berberine}

Biofilm biomass - crystal violet assay. Berberine was screened for its anti-biofilm potential in the pathogenic bacteria using crystal violet microtitre plate assay (CV-MTP) at sub MIC (Fig. 3). Berberine showed significant anti-biofilm potential against both the pathogens. It showed significant inhibitory activity against attachment and biofilm formation in $P$. aeruginosa PA01 at $0.625 \mathrm{mg} \mathrm{mL}^{-1}$ by $71.70 \%$. At $0.019 \mathrm{mg} \mathrm{mL}^{-1}$, it inhibited biofilms of $S$. enterica sv Typhimurium by $31.20 \%$. Thus, it is clear from the above study that berberine was capable of significantly inhibiting bacterial biofilms $(p<0.05)$. Further, the QS inhibitory effect of berberine on P. aeruginosa PA01 JP-2 was studied at $0.078 \mathrm{mg} \mathrm{mL}^{-1}$. The biosensor forms weak biofilms but in presence of AHLs it forms moderate to strong biofilms. However, the addition of berberine reduced the biofilm formation of $P$. aeruginosa PA01 JP-2 in presence of both the signaling molecules C4-HSL and 3-oxo-C12 HSL by 65.50 and $60.74 \%$, respectively (Fig. 3). Thus, the biofilm inhibitory activity of berberine at sub-inhibitory concentrations is due to its anti-quorum sensing property.

\section{Fluorescence microscopy analysis}

Microscopy studies revealed the effect of the phytochemical berberine on bacterial attachment, colonization of surface and 


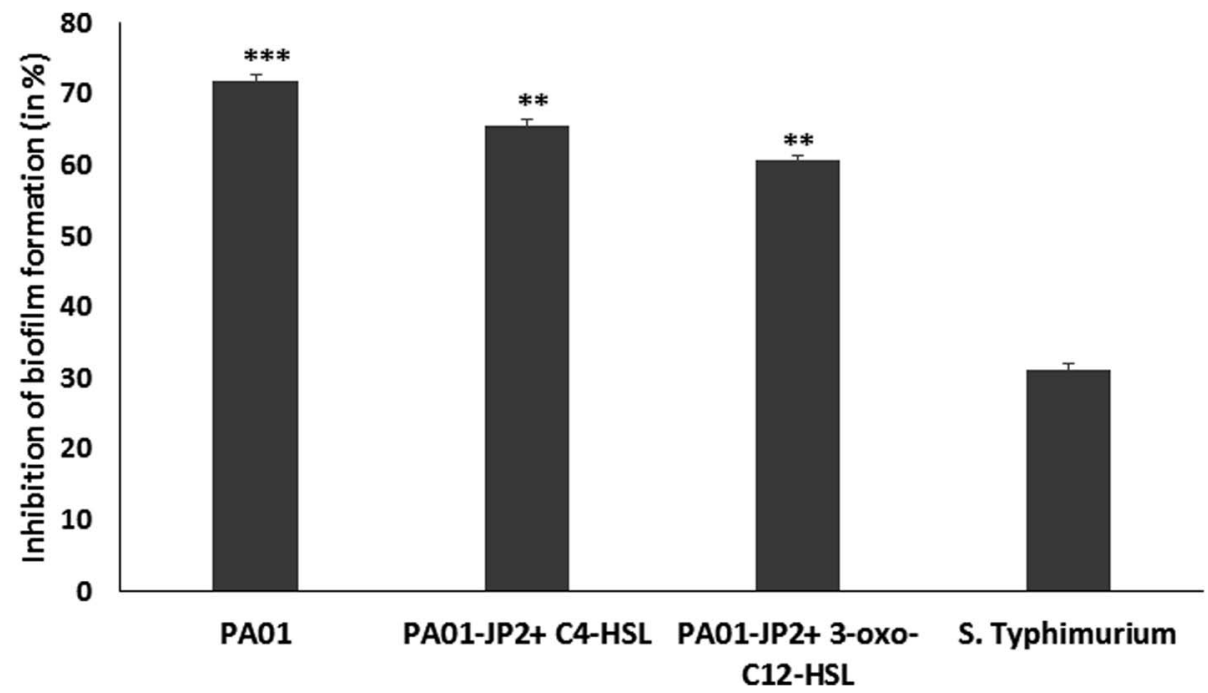

Fig. 3 Anti-biofilm activity of sub-MIC of berberine on P. aeruginosa PA01 (WT and mutant) and S. Typhimurium assessed by crystal violet microtiter plate assay.

formation of mature biofilms. Berberine was able to inhibit attachment of $P$. aeruginosa PA01 cells but at a higher concentration of $0.625 \mathrm{mg} \mathrm{mL}^{-1}$ (Fig. 4). Though berberine was unable to inhibit initial cell attachment in $S$. Typhimurium, however, it was capable of disrupting the pre-formed biofilms. At a concentration of $0.019 \mathrm{mg} \mathrm{mL}^{-1}$, it disrupted colonies of $S$. enterica sv Typhimurium and prevented them from developing into mature biofilms (Fig. 4). Berberine without affecting bacterial growth significantly inhibited biofilm formation in the pathogens by inhibiting adherence to substratum, interfering with EPS production and by reducing metabolic activity leading to prevention of biofilm maturation. Though their activity on
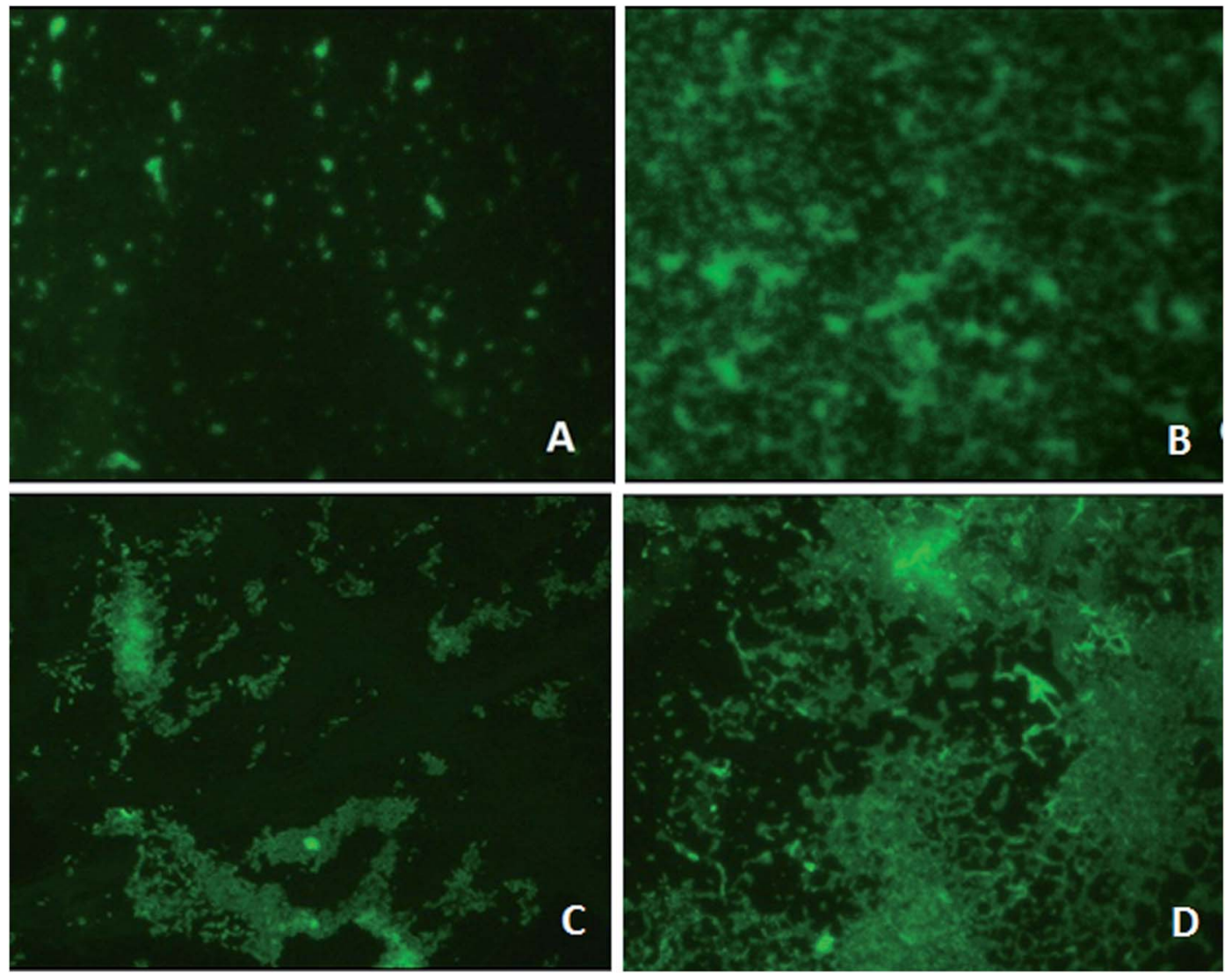

Fig. 4 Fluorescence microscopy analysis of biofilm formation in $P$. aeruginosa in presence of $0.5 \times$ MIC of berberine (A), and untreated control (B); and in S. Typhimurium in presence of $0.5 \times$ MIC of berberine (C), and untreated control (D). 


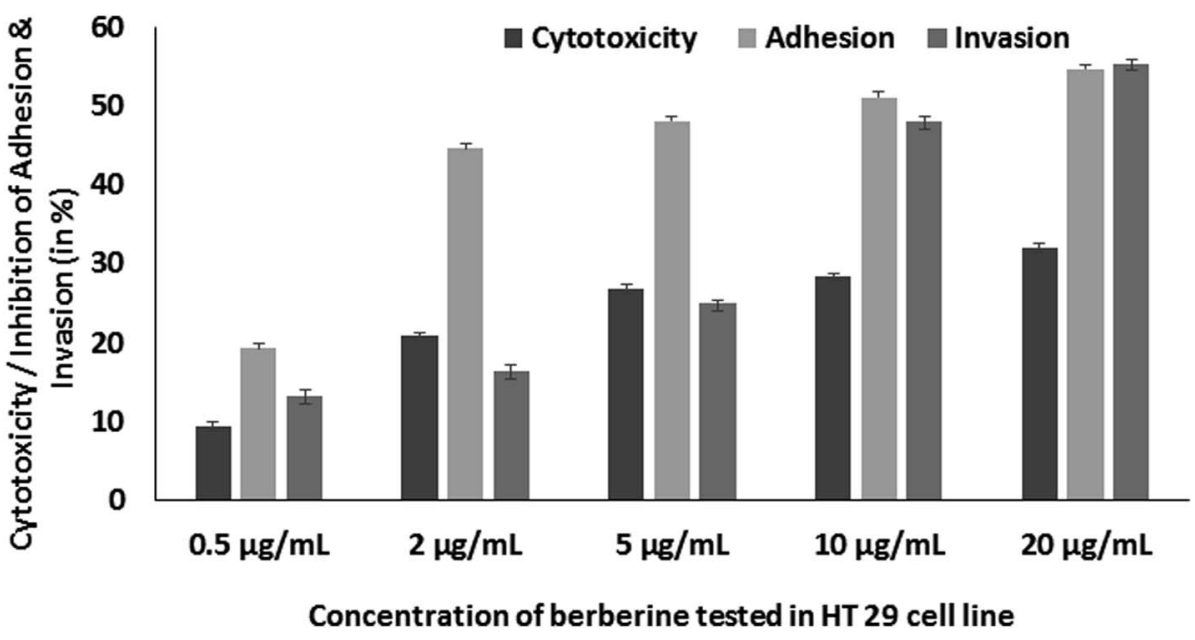

Fig. 5 Concentration dependent effect of berberine on HT 29 cell line cytotoxicity, adhesion and invasion of S. Typhimurium in HT 29 cell line.

initial attachment of $S$. Typhimurium was low, they could disrupt biofilm formation during maturation stage which is probably due to the inhibition of EPS production. Quorum sensing mechanism is known to be involved not only in the initiation but also in the maturation of bacterial biofilms. EPS production in biofilms required for the development of biofilm architecture and maturation has been demonstrated to be under quorum sensing regulon. ${ }^{28}$ Therefore, it can be concluded that the phytochemical berberine at sub-inhibitory concentrations inhibited the attachment of cells to substratum and further colonization of the $P$. aeruginosa and disrupted $S$. Typhimurium biofilms due to its quorum sensing inhibitory potential.

\section{Anti-infective property}

Further, berberine was studied for its anti-virulence property in $S$. Typhimurium. Berberine was tested for cytotoxicity in HT 29 colon cell line by treating it with the phytochemical for $24 \mathrm{~h}$ and measuring the cell viability by MTT assay. Berberine from 0.005 to $0.02 \mathrm{mg} \mathrm{mL}^{-1}$ had no adverse effect on the tested mammalian cell line (Fig. 5). The same concentrations were used to study the effect of berberine on $S$. Typhimurium adhesion and invasion. In a concentration dependent manner, berberine was capable of significantly reducing both adhesion and invasion of $S$. Typhimurium in the mammalian colon cells. At the highest tested concentration of $0.02 \mathrm{mg} \mathrm{mL} \mathrm{m}^{-1}$, berberine reduced adhesion and invasion of $S$. Typhimurium by 54.68 and $55.37 \%$, respectively (Fig. 5). Previously, it has been reported that subinhibitory concentrations of phytochemicals punicalagin and carvacrol significantly reduced Salmonella invasion without affecting adhesion to the intestinal epithelial cells. ${ }^{\mathbf{2 0 , 2 9}}$

C. elegans is used as an animal infection model to study the pathogenicity of bacteria. L4-staged $C$. elegans were infected with $S$. Typhimurium, without or with the addition of berberine and furanone. Infected worms were incubated at $23{ }^{\circ} \mathrm{C}$ for $24 \mathrm{~h}$ and were examined for the infection phenotype. They were observed for paralysed state as neither body twitching nor pharyngeal pumping occurs due to severe infection. C. elegans infected with $S$. Typhimurium showed high paralysis of around $85 \%$, whereas it was observed that berberine and furanone reduced nematode paralysis. Berberine showed a dose dependent reduction in C. elegans infection. At $0.038 \mathrm{mg} \mathrm{mL}^{-1}$, it reduced nematode paralysis by $65.38 \%(p<0.05)$. Furanone, a QS inhibitor showed significant reduction in $S$. Typhimurium induced nematode paralysis and infection (Fig. 6).

S. Typhimurium pathogenesis depends upon a number of factors including virulence factor production, invasion into host and intracellular proliferation. Therefore, alternative strategies such as use of anti-virulence agents, anti-infectives and anti-pathogenic drugs have to be used in the pathogen control. As these agents are used at sub-inhibitory concentration, they impose less selective power for resistance among pathogens.

\section{Molecular docking}

Docking studies were performed to screen the interaction of berberine with the quorum sensing receptors. The receptors after binding with the signals act as transcriptional regulators. The phytochemical were docked against the quorum sensing signal receptors LasR and RhlR (Fig. 7). The docking analysis revealed that had better interaction with the RhlR receptors than the LasR protein as shown by the docking scores and the interaction between the ligand and the receptors (Table 2).

The leucine (L125), tyrosine (Y56), threonine (T75) and asparagine (N73) of LasR and leucine (L116), phenylalanine (F101), tyrosine (Y64), leucine (L85) of RhlR, interacted with berberine. Thus, the amino acids which were involved in bond formation to provide efficient interaction of the ligands with the receptors was identified using the docking analysis. Therefore, by the docking studies it can be concluded that the phytochemical berberine can interact with the quorum sensing receptors. This phytochemical can be used as the lead molecule for the development of new drugs to inhibit quorum sensing in LuxR homologues utilizing pathogenic bacteria.

Thus, in silico studies based on molecular docking analysis have supported the quorum sensing inhibitory behavior of 


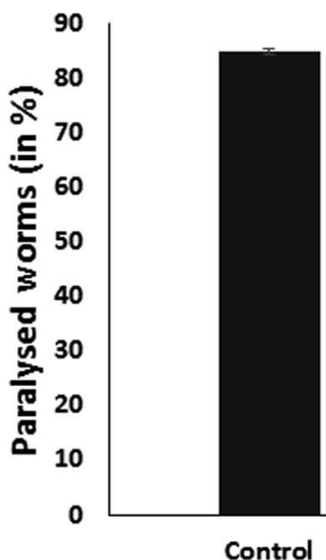

\section{C. elegans infection assay}

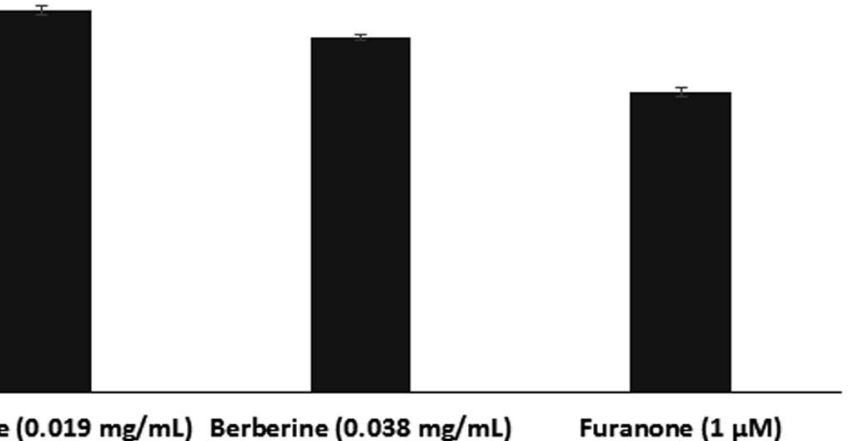

Fig. 6 Effect on berberine $\left(0.019 \mathrm{mg} \mathrm{mL}^{-1}\right.$ and $\left.0.038 \mathrm{mg} \mathrm{mL}^{-1}\right)$ and synthetic QS inhibitor furanone $(1 \mu \mathrm{M})$ on $S$. Typhimurium infection in $C$. elegans animal model evaluated by decrease in number of paralysed worms.

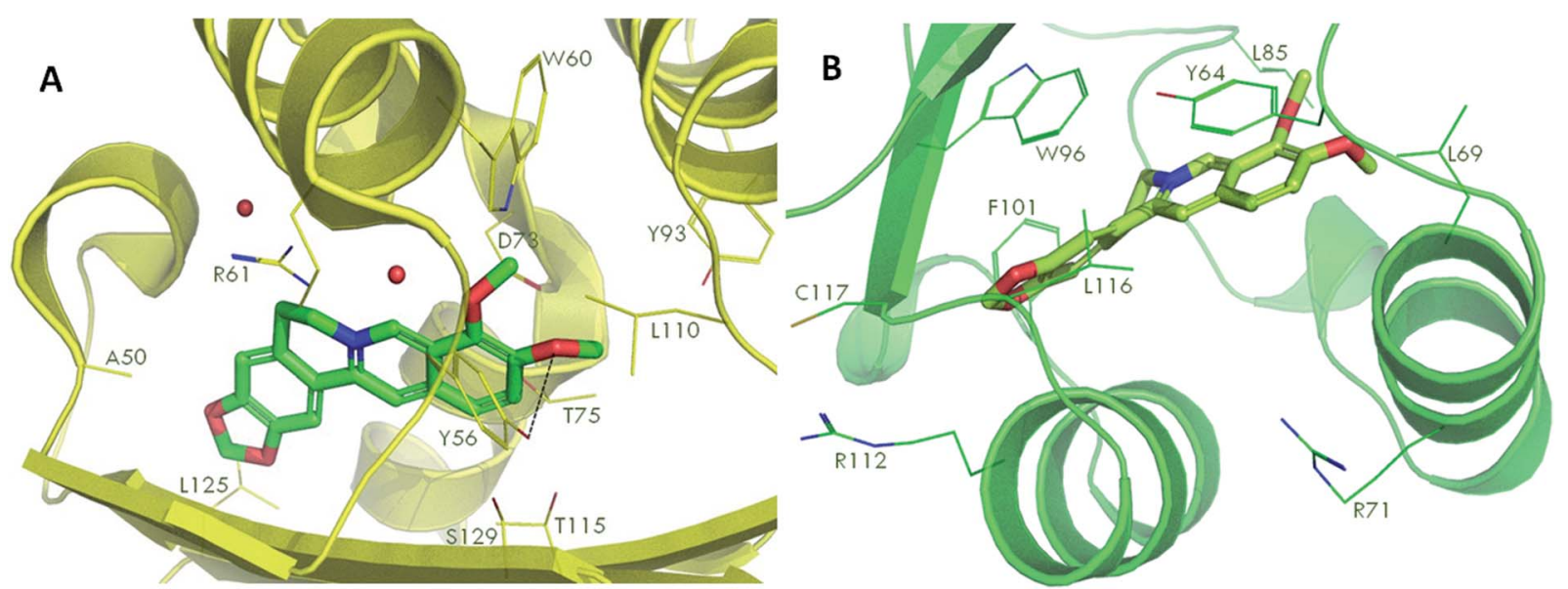

Fig. 7 Molecular docking of phytochemical berberine with QS signal receptors, LasR (A) and RhIR (B).

Table 2 Molecular docking analysis of the phytochemicals with the RhIR and LasR receptors

Glide $6.2^{b}$ extra precision (XP) docking scores

Compound $^{a}$

P. aeruginosa $\mathrm{RhlR}^{c}$

P. aeruginosa $\mathrm{LasR}^{d}$

Berberine

$-10.1$

$-5.9$

${ }^{a}$ Structures downloaded from PubChem. ${ }^{b}$ Molecular docking software (Product of Schrödinger LLC). ${ }^{c}$ Docked in an in-house generated homology model (template: PDB ID - 4LFU, sequence identity 40\%). ${ }^{d}$ Docked in PDB ID - 2UV0, chain H (Quorum sensing signaling molecule binding site).

berberine. In our study, docking analysis revealed the possible interactions between berberine and the QS receptors. Similarly, in $P$. aeruginosa PAO1 LasR and RhlR are receptors for the quorum sensing signal molecules 3oxo-C12 HSL and C4 HSL, respectively. Docking studies were used to explore the probable active sites in these transcriptional regulators with berberine. The overall docking results of the phytochemical with the receptors revealed that the conserved residues tyrosine, leucine, arginine, threonine, asparaginine and phenylalanine were involved in bond formation with the phytochemical. Berberine had strong interaction with the signal receptors which might be the reason for their quorum sensing modulatory property as exhibited by them in the inhibition of violacein production in $C$. violaceum and in disrupting motility and biofilms of $P$. aeruginosa.

Berberine is an isoquinolone alkaloid present in plants of the genus Coptis, Hydrastis and Berberis and these plants have been consumed as a food source and used for their medicinal properties. $^{30}$ The present study, demonstrates that berberine has biofilm inhibitory and anti-quorum sensing property, apart from other therapeutic potentials. 


\section{Conclusion}

The phytochemical berberine at sub-inhibitory concentration was capable of modulating quorum sensing regulated phenotypes and inhibited biofilm formation in the QS biosensor organisms $C$. violaceum and $P$. aeruginosa PA01 as determined by in vitro and in silico analysis. Further, berberine was capable of reducing adhesion, invasion and infection in $S$. Typhimurium. Thus, berberine could find potential application as antiinfective agents to control $P$. aeruginosa and $S$. Typhimurium infections.

\section{Conflicts of interest}

There are no conflicts to declare.

\section{Acknowledgements}

The authors thank Prof. Kalai Mathee, Florida International University, USA for providing the biosensor strains used in the study and CGC Centre, University of Minnesota for C. elegans nematode and $E$. coli OP50. The authors thank Indian Council of Medical Research for awarding fellowship to the first author (Grant No. F.No. 80/709/2011-ECD I). The authors acknowledge Institution of Excellence, University of Mysore for providing fluorescence microscopy imaging facility and DST-PURSE, University of Mysore for Cell culture facility.

\section{References}

1 M. R. Parsek and E. P. Greenberg, Trends Microbiol., 2005, 13, 27-33.

2 S. Asad and S. M. Opal, Crit. Care, 2008, 12, 236-246.

3 M. Simoes, L. C. Simoes and M. J. Vieira, LWT-Food Sci. Technol., 2010, 43, 573-583.

4 U. M. Pinto, V. E. de Souza, M. L. Martins and M. C. D. Vanetti, Food Control, 2007, 18, 1322-1327.

5 M. Teplitski, U. Mathesius and K. P. Rambaugh, Chem. Rev., 2011, 11, 100-116.

6 B. Girennavar, M. L. Cepeda, K. A. Soni, A. Vikram, P. Jesudhasan and G. K. Jayaprakasha, Int. J. Food Microbiol., 2008, 125, 204-208.

7 J. G. Chung, L. T. Wu, C. B. Chu, J. Y. Jan, C. C. Ho, M. F. Tsou, H. F. Lu, G. W. Chen, J. G. Lin and T. F. Wang, Food Chem. Toxicol., 1999, 37, 319-326.

8 M. Chu, M. B. Zhang, Y. C. Liu, J. R. Kang, Z. Y. Chu, K. L. Yin, L. Y. Ding, R. Ding, R. X. Xiao, Y. N. Yin, X. Y. Liu and Y. D. Wang, Sci. Rep., 2016, 6, 24748.
9 X. Wang, X. Yao, Z. Zhu, T. Tang, K. Dai, I. Sadovskaya, S. Flahaut and S. Jabbouri, Int. J. Antimicrob. Agents, 2009, 34, 60-66.

10 N. Guo, X. Zhao, W. Li, C. Shi, R. Meng, Z. Liu and L. Yu, J. Med. Microbiol., 2015, 64, 891-900.

11 L. Chen, Q. Bu, H. Xu, Y. Liu, P. She, R. Tan and Y. Wu, Microbiol. Res., 2016, 186-187, 44-51.

12 Q. Xie, B. R. Johnson, C. S. Wenckus, M. I. Fayad and C. D. Wu, J. Endod., 2012, 38, 1114-1117.

13 S. Jadhav, R. Shah, M. Bhave and E. A. Palombo, Food Control, 2013, 29, 125-130.

14 R. S. Blosser and K. M. Gray, J. Microbiol. Methods, 2004, 40, 47-55.

15 C. O'May and N. Tufenkji, Appl. Environ. Microbiol., 2011, 77, 3061-3067.

16 M. Sandasi, C. M. Leonard and A. M. Viljoen, Lett. Appl. Microbiol., 2010, 50, 30-35.

17 M. O. Vandeputte, M. Kiendrebeogo, S. Rajaonson, B. Diallo, A. Mol, M. E. Jaziri and M. Baucher, Appl. Environ. Microbiol., 2010, 76, 243-253.

18 E. Peeters, H. J. Nelis and T. Coenye, J. Microbiol. Methods, 2008, 72, 157-165.

19 J. McCauley, A. Zivanovic and D. Skropeta, Methods Mol. Biol., 2013, 1055, 191-205.

20 G. Li, C. Yan, Y. Xu, Y. Feng, Q. Wu, X. Lv, B. Yang, X. Wang and X. Xiaa, Appl. Environ. Microbiol., 2014, 80, 6204-6211.

21 S. Puiac, X. Sem, A. Negrea and M. Rhen, Int. J. Antimicrob. Agents, 2011, 38, 409-416.

22 S. Gnanendra, S. Anusuya and J. Natarajan, J. Mol. Model., 2012, 18, 4709-4719.

23 L. R. Swem, D. L. Swem, C. T. O'Loughlin, R. Gatmaitan, B. Zhao, S. M. Ulrich and B. L. Bassler, Mol. Cell, 2009, 35, 143-153.

24 C. Niu, S. Afre and E. S. Gilbert, Lett. Appl. Microbiol., 2006, 43, 489-494.

25 I. A. S. V. Packiavathy, P. Agilandeswari, K. S. Musthafa, S. K. Pandian and A. V. Ravi, Food Res. Int., 2012, 45, 85-92.

26 D. A. Vattem, K. K. Mihali, S. H. Crixell and R. J. Mclean, Fitoterapia, 2007, 78, 302-310.

27 M. Givskov, R. de Nys and M. Manefield, J. Bacteriol., 1996, 178, 6618-6622.

28 T. R. De Kievit, R. Gillis, S. Marx, C. Brown and B. H. Iglewski, Appl. Environ. Microbiol., 2001, 67, 1865-1873.

29 J. Inamuco, A. K. Veenendaal, S. A. Burt, J. A. Post, J. L. Tjeerdsma-van Bokhoven, H. P. Haagsman and E. J. Veldhuizen, Vet. Microbiol., 2012, 157, 200-207.

30 Y. L. Siow, L. Sarna and O. Karmin, Food Res. Int., 2011, 44, 2409-2417. 